\title{
A Real-Time Structural Damage Detection method for High-Pile Wharf Foundations
}

\author{
Yongcheng Liu ${ }^{1, *}$, Yonglai Zheng ${ }^{1}$, and Yujue Zhou ${ }^{1}$ \\ ${ }^{1}$ College of Civil Engineering, Tongji University, Shanghai 200092, China
}

\begin{abstract}
As one of the most common structural forms in port engineering, the operation environment of high-pile wharf is quite harsh and complex, and its pile foundation often produces structural damage of different degrees. Until now, there is a lack of efficient, safe and economic damage detection methods. A novel and precise real-time structural damage detection (SDD) method using both finite element modelling (FEM) and 1D convolutional neural networks (CNNs) is established in this study. The results indicate that the proposed method could accurately identify the presence and location of damage in real time. The results also demonstrated that the proposed 1D CNNs based model are more sensitive to the longitudinal and lateral displacement responses of the high-pile wharf structure.
\end{abstract}

\section{Introduction}

High-pile wharfs need to be monitored in real time to improve their operational performance, prolong their expected life spans, and prevent sudden failures. However, conventional structural damage detection (SDD) methods for the pile foundations of wharfs are laborious and costly[1-3]. Therefore, efficiently detecting and precisely locating the structural damage in foundations have always been formidable challenges.

Studies have shown that vibration-based SDD methods have been adopted by most structural health monitoring (SHM) systems[3-7]. The basic premise of such methods is that the physical properties, such as mass, damping, and stiffness, would change due to damage $[1,2$, $6,8]$. Convolutional neural networks (CNNs), as a type of multilayer feedforward neural network, are extensively used in various supervised classification tasks. However, a large number of the samples in the field of SDD are time series, such as vibration and strain history. Therefore, 1D CNNs have become a state-of-the-art technique to address vibration-based SDD issues in civil structures.

The primary aim of this research is to establish a novel method for the vibration-based SDD of high-pile wharf foundations using FEM and 1D CNNs, which realizes the accurate identification of the existence and location of structural damage. Moreover, we also verify the directional sensitivity of the model to the displacement responses of typical high-pile wharfs, which can be utilized to optimize the acquisition strategy of structural damage dynamic response data under limited sensor conditions.

\section{Methodologies}

\subsection{Geometric model}

A structural sediment in the middle of the high-pile wharf, which is located in Shanghai Port, was chosen for this study as the analysis object. It consisted of 7 lateral bends of which the spacing was $7 \mathrm{~m}$, and each frame was composed of 5 vertical piles and 1 inclined pile. These pile foundations are all square piles with a side length of 600 $\mathrm{mm}$. The main upper structure adopts inverted T-shaped beams and panels that are both made of cast-in-place reinforced concrete. The lower beam is $0.9 \mathrm{~m}$ wide and 0.8 $\mathrm{m}$ high, the upper beam is $0.4 \mathrm{~m}$ wide and $1.6 \mathrm{~m}$ high, the longitudinal beam is $0.4 \mathrm{~m}$ wide and $1.6 \mathrm{~m}$ high, and the plate thickness is $0.8 \mathrm{~m}$.

The " $\mathrm{m}$ " method was utilized to determine the calculated length of the piles to meet the equivalent lateral stiffness principle. The " $\mathrm{m}$ " method assumes that the horizontal foundation resistance coefficient of soil increases linearly with the depth of soil, namely:

$$
\mathrm{K}=\mathrm{mz}
$$

where $\mathrm{K}$ denotes the horizontal foundation resistance coefficient of soil $\left(\mathrm{kN} / \mathrm{m}^{3}\right), \mathrm{m}$ is the proportional coefficient $\left(\mathrm{kN} / \mathrm{m}^{4}\right)$, and $\mathrm{z}$ represents the depth of the calculated point.

Since the original foundation soil is distributed in layers, the m-value adopts the weighted average of $\mathrm{m}$ of each soil layer at a depth of $1.8 \mathrm{~T}$ below the ground:

$$
T=\sqrt[5]{\frac{E_{P} I_{P}}{m b_{0}}}
$$

where $E_{p}$ indicates the modulus of elasticity of piles 
$\left(\mathrm{kN} / \mathrm{m}^{2}\right), I_{p}$ is the cross-sectional moment of the inertia of piles $\left(\mathrm{m}^{4}\right)$, and $b_{0}$ is the converted width (m). $b_{0}$ is calculated as:

$$
\mathrm{b}_{0}=\mathrm{k}_{\mathrm{f}}(1.5 \mathrm{~d}+0.5)
$$

where $k_{f}$ is the shape conversion factor of piles. Here, this value is set as 1.0.

The structural foundation square piles were prefabricated using C30 concrete, and the modulus of elasticity was $2.7 \mathrm{e} 4 \mathrm{MPa}$. Moreover, the straight piles are $31 \mathrm{~m}$ long, the oblique piles are $32 \mathrm{~m}$ long, and the maximum depth of piles is $18.25 \mathrm{~m}$.

The depth of the embedded point is calculated according to the following equation:

$$
\mathrm{t}=\eta \mathrm{T}
$$

where the empirical parameter takes values ranging from 1.8 to 2.2. This study sets the parameter as 2.0.

The calculated length of each piles are listed in Table 1 as follows.

Table 1. Summary of the calculation results of the relative depth of each pile bottom embedded point to the pile top based on the "m" method (m)

\begin{tabular}{|c|c|c|c|c|c|c|}
\hline $\begin{array}{c}\text { Pile } \\
\text { Number }\end{array}$ & 1 & 2 & 3 & 4 & 5 & 6 \\
\hline $\begin{array}{c}\text { Calculated } \\
\text { Length }\end{array}$ & 14.4 & 13 & 11.4 & 12.4 & 9.8 & 8.55 \\
\hline
\end{tabular}

\subsection{Structural damage scheme}

The essence of structural damage can be defined as the loss of local stiffness in the damaged section. The structural sediment selected by this research has a total of 42 foundation piles, which were numbered sequentially from the top left to the bottom right of the structural floor plan. Condition $0^{\#}$ means that none of the piles have reduced stiffness, similarly, condition $i^{\#}$ means that pile $i^{\#}$ has its stiffness reduced $50 \%$ to simulate the global stiffness degradation induced by structural damage.

\subsection{Finite element model simulation}

The FE model of the structural sediment of a high-pile wharf was built in ANSYS 19.0. The interactions between pile foundations and soil were simplified, and fixed constraints were imposed on the bottom of all pile foundations. The grid resolution of $100 \mathrm{~mm}$ is used in this research to perform the transient dynamics calculation of the FE model.

\section{The proposed One-Dimension CNNs}

\section{1 overall architecture}

The proposed 1D CNN architecture in this paper contains 12 hidden layers, specifically, 6 1D convolutional layers,
2 local pooling layers, 2 batch normalization layers, 1 global pooling layer and 1 random deactivation (dropout) layer.

\section{2 data preprocessing}

In this study, displacement dataset in component form was constructed to evaluate the performance of the $\mathrm{CNN}$ models. For each damage condition, the displacement responses of all 42 beam-pile nodes in three directions were horizontally spliced. Next, we add a column along the end of the tensor as a label, and the content of the column is the current damage condition number $i$ ( $i$ is an integer from 0 to 43 ). At this time, the displacement response data under each condition are formed in a $2 \mathrm{D}$ tensor format with dimensions of $(2494,127)$. Finally, the data of all damage conditions are vertically spliced according to the principle of measurement point alignment, and a $2 \mathrm{D}$ tensor $D_{c}$ with dimensions of $(107242,127)$ is constructed. Each label item of 214484 samples of the training datasets $D_{c}$, were all recoded as a $1 \mathrm{D}$ tensor with a dimension of $(43,1)$ by the one-hot coding principle.

\subsection{The training setup}

Based on the idea of orthogonal experiments, this study established the following training strategies, as shown in Table 2. Briefly, in each training group, the population samples were randomly divided into $80 \%, 10 \%$ and $10 \%$, as the training set, validation set, and testing set, respectively. The initial value of learning rate was 0.0001 .

Table 2. Setup of the training groups

\begin{tabular}{|c|c|c|c|}
\hline $\begin{array}{c}\text { Group } \\
\text { number }\end{array}$ & \multicolumn{3}{|c|}{ Direction of displacement response } \\
\hline & $\begin{array}{c}\text { Longitudinal } \\
\text { direction }\end{array}$ & $\begin{array}{c}\text { Vertical } \\
\text { direction }\end{array}$ & $\begin{array}{c}\text { Transverse } \\
\text { direction }\end{array}$ \\
\hline 1 & $\bullet$ & & \\
\hline 2 & & $\bullet$ & \\
\hline 3 & & & $\bullet$ \\
\hline 4 & $\bullet$ & $\bullet$ & \\
\hline 5 & & $\bullet$ & $\bullet$ \\
\hline 6 & $\bullet$ & & $\bullet$ \\
\hline
\end{tabular}

\section{Results and discussions}

\subsection{Impact of data format on model performance}

The models have demonstrated superior recognition accuracy performance $(93.4 \%)$ over the course of 100 training epochs. The Figure 1 shows that in the first 20 training epochs, the training accuracy of the model increased rapidly as the number of iterations increased. After more than 70 training epochs, the growth trend of the training accuracy is very slow and gradually levels off. Moreover, in the first 60 training epochs, the validation accuracy of the model is highly consistent with the trend of the training accuracy. 


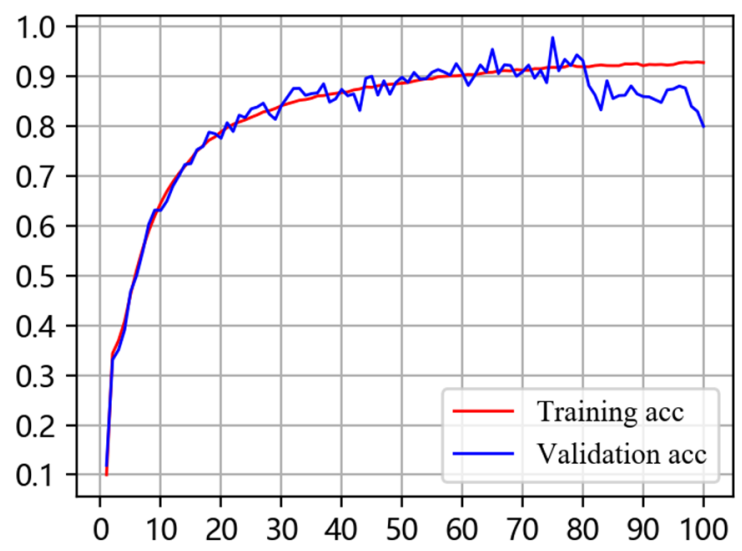

Figure 1. Model performance of the $1 \mathrm{D}$ CNNs based on dataset in component form
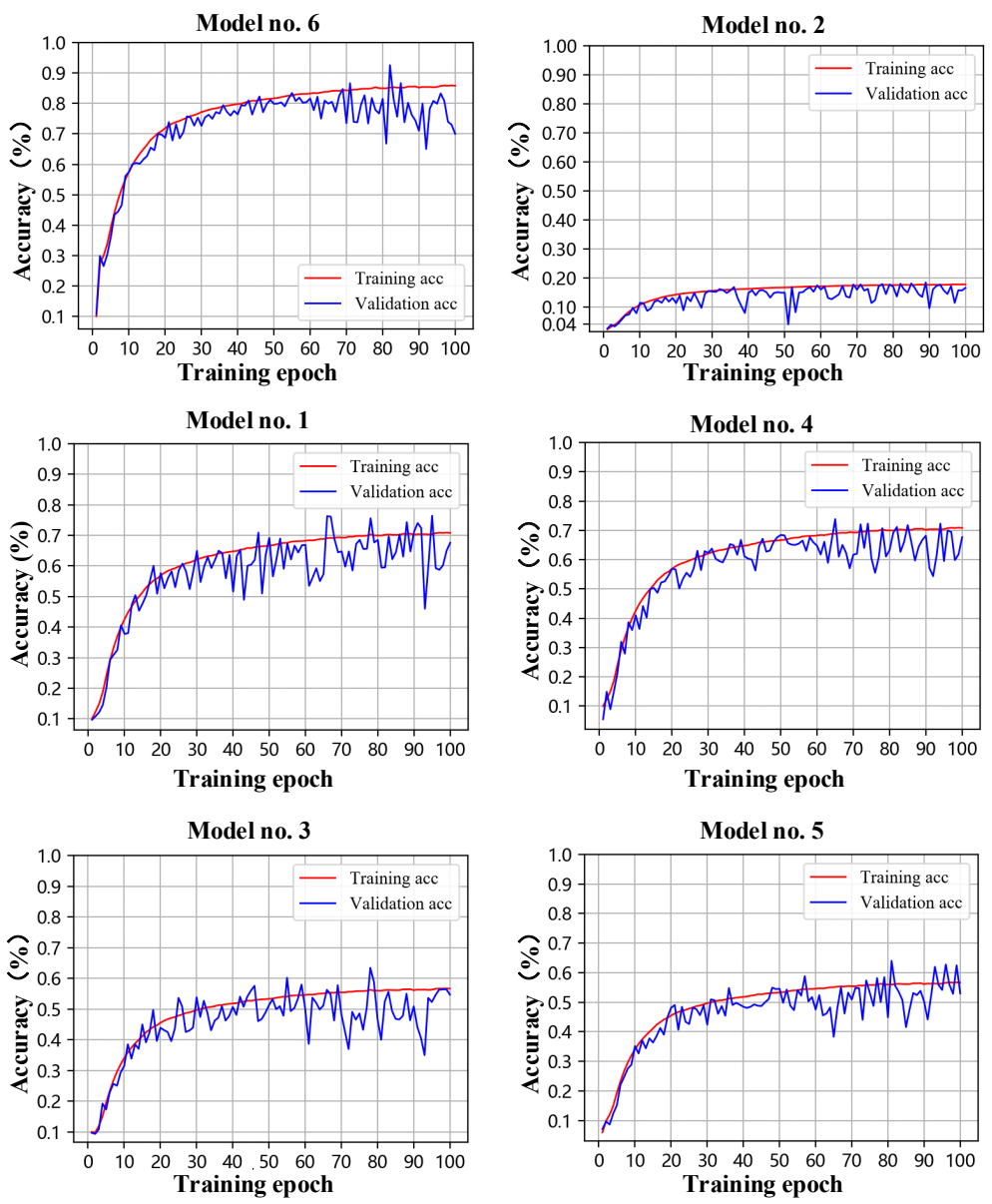

Figure 2. Performance of the 1D CNN model based on 6 groups of reduced component displacement response datasets

\subsection{Directional sensitivity of the model to the displacement response}

The training accuracy and validation accuracy of the above 6 models are shown in Figure 2. Several main results can be summarized as follows:

(a) The classification accuracies of the model trained by training group no. 6 are slightly lower than those of the model based on the dataset in component form. This indicates that the vertical displacement responses of the structure have very limited impacts on the damage identification accuracy.

(b) The classification accuracies of model no. 4 and model no. 1 were significantly higher than those of model no. 5 and model no. 3 , implying that the longitudinal displacement response component outweighs the lateral displacement response component.

(c) The validation accuracy of the model no. 6 was significantly more discrete than that of the model based on the dataset in component form. Furthermore, the generalization abilities of model no. 4 and model no. 5 significantly outperform those of model no. 1 and model no. 3 . These phenomena further confirm that the vertical displacement response components of the structural segment of the high-pile wharf have an effect on improving the generalization and robustness of the 1D CNN model. 


\section{Conclusions}

This study applies a 1D CNN-based approach to the vibration-based structural damage detection of typical high-pile wharf foundations. The results indicated the superior performance of the $1 \mathrm{D} \mathrm{CNNs}$ to extract the damage-sensitive features directly from the raw displacement response data.

The " $m$ " method, which calculates the depth of the embedding point of the pile, is shown to be useful for simplified structural modeling. Besides, the sample datasets required for the training of the proposed CNNs can be directly reconstructed by the displacement response time series of all the beam-pile nodes of the FE model.

The proposed CNNs are more sensitive to the longitudinal and lateral displacement responses at the beam-pile nodes. More specifically, the former has a higher contribution to the classification accuracy of CNNs than the latter and their effects both significantly outweigh the influence of vertical displacement. Although the contribution of the vertical displacement response to the improvement of the model identification accuracy is rather limited, this component has a positive effect on the improvement of the generalization and robustness of the CNNs.

\section{Acknowledgments}

This work was supported by the major projects of Science and Technology Commission of Shanghai Municipality, China (17DZ1202904).

\section{References}

1. O. Avci, O. Abdeljaber, S. Kiranyaz, M. Hussein, M. Gabbouj, D. J. Inman, B.R. Ames, IA. Mech. Syst. Signal. Process 147 (2021)

2. S. Das, P. Saha, S. K. Patro, Kopargaon, Maharashtra, India. J. Civ Struct. Health. Monit 6 (2016)

3. V. Alves, A. Cury, Juiz de Fora, Brazil. Struct. Control. Health. Monit 8 (2018)

4. Y.J. Yan, L. Cheng, Z.Y. Wu, L.H. Yam, Xi'an, China. Mech. Syst. Signal. Process 21 (2007)

5. R. Ghiasi, P. Torkzadeh, M. Noori, Kerman, Iran. Struct. Health. Monit 15 (2016)

6. Fan, W. and P. Qiao, SHM, 10(2011) W. Fan, P.Z. Qiao, Pullman, WA, USA. Struct. Health. Monit 10 (2011)

7. R.R. Hou, Y. Xia, Kowloon, Hong Kong, China. J. Sound. Vib 20 (2020)

8. C. R. Farrar, S.W. Doebling, D. A. Nix, R. Soc. Lond. Philos. Trans. A. Math. Phys. Eng. Sci 359 (2001) 